\title{
La formation des médiévistes dans le Brésil contemporain : bilans et perspectives (1985-2007)
}

Néri de Barros Almeida

\section{(2) OpenEdition \\ 1 Journals}

Édition électronique

URL : https://journals.openedition.org/cem/6652

DOI : $10.4000 /$ cem.6652

ISSN : 1954-3093

Éditeur

Centre d'études médiévales Saint-Germain d'Auxerre

Édition imprimée

Date de publication : 15 août 2008

ISSN : 1623-5770

Référence électronique

Néri de Barros Almeida, «La formation des médiévistes dans le Brésil contemporain : bilans et perspectives (1985-2007) », Bulletin du centre d'études médiévales d'Auxerre / BUCEMA [En ligne], 12 | 2008, mis en ligne le 09 avril 2009, consulté le 22 septembre 2022. URL : http:// journals.openedition.org/cem/6652 ; DOI : https://doi.org/10.4000/cem.6652

Ce document a été généré automatiquement le 22 septembre 2022.

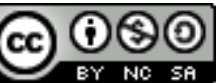

Creative Commons - Attribution - Pas d'Utilisation Commerciale - Partage dans les Mêmes Conditions 4.0 International - CC BY-NC-SA 4.0

https://creativecommons.org/licenses/by-nc-sa/4.0/ 


\title{
La formation des médiévistes dans le Brésil contemporain : bilans et perspectives (1985-2007) *
}

\author{
Néri de Barros Almeida
}

1 L'effort systématique pour former des chercheurs capables de produire des connaissances dans le domaine de l'histoire médiévale au Brésil est très récent. Il date des années 1980. Historiquement, son développement accompagne de près celui de la modernisation récente du système universitaire brésilien. Un bilan très large de la trajectoire suivie par les études sur l'histoire médiévale au Brésil conduit à observer essentiellement deux domaines complémentaires. En premier lieu, celui de l'appui et des directives gouvernementales pour la recherche en général, lesquels sont à l'origine de la réorganisation et de l'encouragement à la spécialisation des champs du savoir et qui ont favorisé l'apparition d'un volume significatif des projets visant l'histoire médiévale. En second lieu, le profil scientifique du projet de constitution des premières universités brésiliennes, à l'origine de l'implantation des bases du système de l'enseignement supérieur et de la recherche de notre pays. Ces deux éléments nous permettent de mieux comprendre la dynamique historique qui a présidé à l'implantation des études en histoire médiévale au Brésil, les raisons du retard qui a affecté l'apparition d'un mouvement continu et quantitativement significatif de travaux académiques dans ce domaine, son propre héritage - tant en ce qui concerne les thèmes, méthodes et positions théoriques de la tradition historiographique brésilienne, que de la tradition européenne, notamment française -, tout autant que ses possibilités futures.

2 Bien que la création des universités précède l'apparition des mécanismes fédéraux de régulation et d'aide à la recherche, nous commencerons par ceux-là, car le développement des études médiévales au Brésil coïncide avec leur épanouissement à partir du milieu des années 1980. Dès lors, ces mécanismes ont engendré une augmentation significative du nombre des recherches et ont facilité par ailleurs la diffusion coordonnée des programmes post-universitaires dans tout le pays. Par la 
suite, nous en reviendrons aux universités, puisque c'est d'elles que procède l'héritage à l'origine directe des thèmes, méthodes et théories qui ont marqué le développement de ces champs scientifiques au Brésil.

3 Les universités furent et continuent d'être les principaux centres de formation de chercheurs et de production scientifique de notre pays. Le développement de la recherche, pour sa part, dépendait de l'organisation des programmes du troisième cycle de l'enseignement supérieur, qui survint tardivement, à partir de 1968, et de l'apport de ressources complémentaires au budget universitaire, à travers les organismes gouvernementaux de soutien à la recherche, créés à partir de 1951.

4 Aujourd'hui, il existe au Brésil deux grandes institutions fédérales ayant pour mission principale de promouvoir la recherche scientifique : le CNPq - initialement Conseil national de la recherche, puis Conseil national du développement scientifique et technologique - et la CAPES - Coordination pour le perfectionnement du personnel de formation supérieure -, ainsi que de nombreuses institutions dans les états fédérés, dont notamment les fondations pour le soutien de la recherche. Parmi celles-ci, la FAPESP - Fondation pour le soutien de la recherche de l'État de São Paulo - se distingue par la capacité et le dynamisme de ses aides, fruit d'une politique qui assure un transfert continu et régulier de ressources en provenance du budget de l'état fédéré le plus riche du Brésil, équivalant actuellement à $1 \%$ de ses recettes.

5 Le premier projet, dont est issu le CNPq, est apparu en 1936, à l'initiative de l'ABC Académie brésilienne des sciences, fondée en 1916 -, dans le cadre de la création de la première université brésilienne, l'université de São Paulo (USP), en 1934. Après 1945, et comme conséquence de l'engouement pour les sciences suscité par la seconde guerre mondiale, les conditions favorables à la création des agences de soutien sont apparues. En 1946, la proposition de création du CNPq est présentée par l'ingénieur amiral Álvaro Alberto Motta e Silva, membre brésilien de la Commission de l'énergie atomique du Conseil de sécurité de l'ONU (Organisation des Nations unies). L'année suivante, l'article 23 de la Constitution de l'État de São Paulo prévoit la création de la FAPESP et l'affectation permanente de $0,5 \%$ des recettes de l'état pour son fonctionnement. Néanmoins, ce n'est qu'en janvier 1951, à la fin du gouvernement du président Dutra, que le CNPq sera finalement créé, avec pour mission de former des ressources humaines pour la recherche - l'accent étant mis sur les sciences de base, comme la physique et la biologie - et d'appuyer le processus d'industrialisation.

6 En juillet 1951, durant le gouvernement du président Vargas, la CAPES est créée, ayant pour mission spécifique d'organiser la mise en œuvre de l'évaluation, de la diffusion des informations et des investissements pour la formation des ressources humaines et de promouvoir la coopération internationale. Par ailleurs, en octobre 1960, la FAPESP est finalement mise en place, ce qui lui permet de débuter ses activités dès 1962. Les premiers pas de ces organisations subissent les effets du populisme des années cinquante, puis, à partir de 1964, ceux de la dictature militaire, laquelle les maintient dans un état de profonde instabilité financière, due au contingentement permanent des ressources qui leur sont allouées. À la fin de la dictature, en 1985, l'hyper-inflation qui sévissait à l'époque continua de compromettre la mise en œuvre des politiques à long terme de soutien à la recherche. Malgré cela, on se doit de reconnaitre que, même s'il ne répondait pas aux besoins croissants et n'accompagnait pas le taux de croissance de la population, le volume des aides accordées s'est accru et que les agences gouvernementales ont peu à peu contribué - soit par l'attribution directe de bourses 
d'études, soit en permettant l'accès à celles-ci - à établir de nouvelles perspectives de production pour la connaissance scientifique.

7 La première année de son fonctionnement, la CAPES a attribué 79 bourses d'études, dont deux bourses de formation au Brésil, 23 bourses de perfectionnement à accomplir au Brésil et 54 à l'étranger. L'année suivante, ce furent 155 bourses, dont 32 de formation au Brésil, 51 de perfectionnement au Brésil et 72 à l'étranger. Engagée, parailleurs, dans l'expansion et la consolidation de la formation en troisième cycle universitaire stricto sensu (master ${ }^{2}$ et doctorat), la CAPES comptait à son actif, en 1965, pour tout le pays, 27 formations de master et 11 de doctorat. Le développement des organismesde soutien à la formation et à la recherche pendant les années de dictature (1964-1985) fut prioritairement axé sur la formation des professionnels de l'industrie et sur le renforcement des dispositifs technologiques liés au projet politique de modernisation. Dans les universités, cette orientation a conduit à définir les disciplines techniques comme étant prioritaires, tandis que dans le domaine des sciences humaines elle s'est traduite par le maintien d'une pratique conservatrice et individualiste pour la détermination des projets de recherche et la sélection des chercheurs débutants. En 1951, le CNPq a attribué 155 bourses d'études, 37 pour les sciences exactes (environ $61 \%$ des ressources), 31 pour les sciences biologiques et appliquées (près de $37 \%$ des ressources), 7 pour des projets divers (environ $1,2 \%$ des ressources). Malgré la disparité de traitement entre les différents domaines de connaissance, une culture de soutien à la recherche donnait ainsi des signes de sa mise en œuvre. De la même manière, les statistiques montrent une augmentation du nombre de formateurs professionnels au Brésil. En 1968, le CNPq offrait déjà 1083 bourses, dont 1009 pour des boursiers accomplissant leur formation au Brésil et 74 pour des formations à l'étranger.

8 L'augmentation progressive des aides connut un revers après 1989, en raison de l'instabilité politique et financière vécue par le pays à cette époque. La même année, le CNPq a accordé 25457 bourses et aides. À partir de 1995, le contrôle de l'inflation, la stabilité institutionnelle et l'allocation régulière et planifiée de ressources ont permis une augmentation consistante des aides et des bourses, ainsi qu'une expansion de la politique de soutien à la recherche. En 1995, le CNPq a accordé52 041 bourses. En 2005, parmi les 1831 bourses accordées par la CAPES, 14,5\% furent destinées au domaine des sciences humaines et parmiles 4306 dossiers étudiés, 119, soit 2,76\% d'entre eux, concernaient l'histoire. Au cours de cette décennie, le domaine de l'histoire médiévale a bénéficié d'une politique qui, pour l'avoir classé parmi les domaines déficitaires du pays, a permis aux projets concernant cette discipline d'accéder en priorité aux aides provenant aussi bien du Brésil que de l'étranger.

9 La CAPES et le CNPq ont fait les premiers pas vers l'institutionnalisation des études de troisième cycle universitaire dans notre pays. Néanmoins, ce ne fut qu'à la fin des années 1960, que le gouvernement militaire, en donnant une impulsion à la politique de modernisation, basée sur le binôme "sécurité et développement ", a mis en œuvre des politiques pour les sciences et la technologie à caractère systématique et global. En 1965, les formations du troisième cycle universitaire sont définies de façon systématique, ce qui leur confère un vrai statut, à l'exemple de celui déjà existant pour la formation universitaire de base - équivalant aux premier et deuxième cycles en France. La loi de la réforme universitaire (loi $n^{\circ} 5.540$ ), promulguée en 1968, tout en incorporant les idées et les expériences de l'université de Brasília (UnB), fondée en 
1961, structure les universités en créant des instituts et des départements, dont la vocation fondamentale est d'assurer à la fois des activités d'enseignement et de recherche.

10 La structuration fédérale des études post-universitaires au Brésil a permis d'organiser la réglementation de la délivrance des titres universitaires, le développement et la spécialisation du système de l'enseignement public supérieur. Dans un premier temps, celui-ci a intégré une grande partie des docteurs déjà formés à l'époque et qui, dans les années 1990, étaient à l'origine des pôles régionaux de préparation au doctorat. Au même moment, des politiques cohérentes et permanentes d'aide à l'initiation scientifique et aux formations au master, au doctorat et au post-doctorat ont assuré l'expansion des recherches.

11 En dépit de fortes disparités régionales, le développement des études postuniversitaires est demeuré stable jusqu'en 1995, date à partir de laquelle il s'est considérablement accéléré. En 1996, 88 établissements d'enseignement supérieur offraient des cours post-universitaires et proposaient au total 1105 programmes (564 pourle master, 22 de doctorat et 519 à la fois pour le master et de doctorat). En 2002, le nombre de ces établissements s'élevait à 148 , proposant 1570 programmes de formation, dont 691 pour le master, 30 pour le doctorat, 784 pour le master et le doctorat, 34 programmes professionnalisants, quatre de master et professionnalisant, 27 à la fois de master, de doctorat et professionnalisant, atteignant, en 2003, le nombre de 1819 programmes. Certes, il reste vrai que, au cours des douze dernières années, une partie importante de cette croissance a été le fruit des réformes gouvernementales de soutien à l'enseignement supérieur privé, tourné, quant à lui, vers les études postuniversitaires lato sensu et la spécialisation professionnelle et non pas vers la recherche.

12 La stabilité des années 1990 a permis l'apparition de nouvelles modalités d'aide, tels les "projets thématiques " et les initiatives de recherche collective pluri-institutionnelle d'un coût élevé, qui associent des chercheurs au sein d'un projet commun. Il s'agit là d'une démarche qui exerce des pressions pour que les bases individualistes des projets universitaires adoptent un nouveau profil, ce qu'elle fait en privilégiantla structuration d'associations de recherche de différents types, comme les groupes, les laboratoires et les centres de recherche spécialisés. Nous pouvons affirmer qu'au cours des quinze dernières années les gouvernements successifs ont mené sans discontinuer des politiques d'investissement dans la recherche bien coordonnées et cohérentes. Les nouvelles initiatives qui ont modifié peu à peu le profil de la recherche universitaire ont été parfaitement soutenues par des modalités d'aide institutionnelles, qui ont permis la réalisation de programmes d'infrastructure, l'installation et la modernisation de laboratoires, l'importation d'équipements coûteux, la visite de professeurs étrangers pour des séjours de longue durée, des bourses spéciales pour que des docteurs nouvellement diplômés puissent exercer leur activité d'enseignant au moins trois ans, des bourses post-doctorales qui permettent à ces docteurs de conduire de nouveaux projets de recherche, tout en exerçant leur activité d'enseignant, des achats massifs de livres, parmi lesquels on distinguera le programme de la FAPESP, connu sous l'appellation de « Fap-livres » ${ }^{3}$.

13 La démocratisation progressive de l'accès à l'enseignement supérieur s'est traduite par l'augmentation de sa capacité d'accueil et du nombre des universités privées et publiques. Ce processus, en modifiant le profil des universités a permis de rompre avec les initiatives isolées quicaractérisaientjusqu'alorsla recherchescientifique. 
L'organisation des études post-universitaires en domaines distincts - histoire sociale, histoire économique, histoire politique, histoire culturelle - a mis en évidence les recherches conduites dans le cadre des différentes disciplines - histoire ancienne, histoire moderne, histoire contemporaine, histoire du Brésil, histoire des Amériques, histoire de l'Afrique, etc. - et stimulé leur regroupement. Enfin, l'augmentation du nombre de postes offerts par les universités publiques dans les dix dernières années, ajoutée aux retraites anticipées en raison des changements survenus dans la législation de la sécurité sociale, a favorisé la mise en place rapide dans les universités d'une nouvelle génération d'enseignants, formée dans un contexte intégrant les valeurs de la recherche collective et les critères internationaux de productivité implantés par la CAPES.

Outre les cours post-universitaires, l'ensemble des laboratoires, groupes et centres de recherche, rattachés aux universités, joue aujourd'hui un rôle majeur dans la formation des spécialistes en histoire médiévale et dans le lancement des projets destinés au développement et au perfectionnement de ce domaine d'études. Citons notamment: l'organisation de manifestations et publications spécifiques, l'élaboration de conventions et d'accords internationaux, la préparation de cours avec des professeurs invités, la promotion des doctorats dits "pleins ", c'est-à-dire, qui sont entièrement réalisés dans un pays étranger, l'organisation de manifestations et de recherches thématiques, la coordination des efforts pour l'obtention de ressources d'infrastructure (livres et équipements), etc. Parmi les groupes qui mènent aujourd'hui une action visant à perfectionner la recherche en histoire médiévale, mentionnons notamment : le Programa de Estudos Medievais (Programme d'études médiévales, PEM) de l'université de Brasília (UnB) et de l'université fédérale de Goiás (UFGO), lequel existe depuis 1994, coordonné par Maria Eurydice de Barros Ribeiro; le Scriptorium - Laboratório de Estudos Medievais e Ibéricos de l'Université Fédérale Fluminense (UFF), dans l'État de Rio de Janeiro, coordonné par Vânia Leite Fróes ; et le Programa de Estudos Medievais (PEM) de l'université fédérale de Rio de Janeiro (UFRJ), coordonné par Andréia Cristina Frazão da Silva et Leila Rodrigues da Silva; à São Paulo, le Laboratório de Estudos Medievais (LEME), une initiative lancée en commun par l'université de São Paulo (USP) et l'université Estadual de Campinas (UNICAMP), la coordination étant assurée par Marcelo Cândido da Silva et moi-même. Tous ces groupes, nés dans les années 1990, sont le résultatde la multiplication des centres d'études post-universitaires au Brésil. Ainsi, l'université de São Paulo ne détient plus le monopolede la formation des docteurs en histoire médiévale, comme c'était le cas jusqu'à la fin des années 1980. À partir des années 1990, la formation de ces professionnels, en dehors de l'État de São Paulo, inclut désormais les programmes d'études post-universitaires de l'université fédérale Fluminense et de l'université fédérale de Rio de Janeiro. Au cours des sept dernières années, les centres de formation ont proliféré dans les villes de l'intérieur de l'État de São Paulo (Campinas, Franca, Assis), ainsi que dans d'autres états fédérés du Brésil : Goiás, Paraná, Rio Grande do Sul, Minas Gerais, Espírito Santo, etc. ${ }^{4}$.

Cette expansion générale a conduit, ces dernières années, l'Associação Nacional de História (ANPUH) - organisme responsable du débat institutionnel sur les politiques publiques d'enseignement et de recherche en histoire, créé en 1961 - à reformuler le mode de réunion de ses membres à l'occasion des symposiums nationaux et des rencontres régionales biennales. Ceux-ci sont désormais organisés en groupes de recherche responsable d'un projet bien déterminé et doivent présenter des propositions de résultats à moyen terme. Aujourd'hui, l'ANPUH organise trois 
symposiums thématiques nationaux, réunissant des chercheurs qui se consacrent à la recherche en histoire médiévale: l'un est coordonné par Vânia Leite Fróes (UFF), l'autre par Andréa Cristina Lopes Frazão da Silva (UERJ) en compagnie de Rejane Barreto Jardim (université de Caxias do Sul) et j'assure, pour ma part, la coordination du troisième (UNICAMP), avec Marcelo Cândido da Silva (USP), lequel met en œuvre le projet « Dualidade de foros et subjetividade política no Ocidente medieval " ${ }^{5}$. L'ANPUH a également été responsable de la création de pôles de recherche régionaux, tel le Grupo de Trabalho de Estudos Medievais, de l'université fédérale du Rio Grande do Sul, qui se réunit tous les mois pour discuter des projets et des thèmes de recherche et, par ailleurs, réaliser périodiquement des manifestations universitaires.

Ces groupes concentrent leurs efforts sur la résolution des grands problèmes de ces champs scientifiques : en premier lieu, la mise en place d'échanges universitaires entre institutions par le biais de l'association pour le développement des projets de recherche, la promotion d'une meilleure utilisation des ressources bibliographiques disponibles, mesure essentielle dans un pays aux dimensions continentales comme le nôtre, et l'organisation des moyens de perfectionnement techniques, au moyen de la réalisation, entre autres, de cours spéciaux. En second lieu, les réunions de ANPUH permettent la divulgation et la discussion au niveau national de projets menés au sein des universités, ainsi qu'une meilleure visibilité institutionnelle de ce domaine, lors des réunions, qui étudient les principales propositions pour le développement de l'enseignement et de la recherche en histoire destinées à être présentées au gouvernement fédéral.

17 La diffusion au Brésil de la production traitant du Moyen Âge progresse d'année en année. L'augmentation du nombre de revues scientifiques dans notre pays (1 882 en 2001 et 9400 en 2005) a permis d'assurer une place chaque fois plus importante aux dossiers et articles touchant à l'histoire médiévale. Au Brésil, la revue Signum de l'ABREM (Association brésilienne d'études médiévales) a su maintenir une bonne qualité en traduisant des articles considérés comme des classiques et en publiant les textes d'auteurs invités. Liée à une association qui réunit des gens intéressés de manière générale par les études concernant l'histoire, la philosophie et la littérature du Moyen Âge, la revue souffre malheureusement d'une faible périodicité (un numéro par an), ce qui empêche son soutien par des institutions publiques. En conséquence, son tirage est modeste, sa distribution est restreinte aux membres de l'association, et même les bibliothèques universitaires peinent à se la procurer.

Quoi qu'il en soit, les grandes revues scientifiques spécialisées en histoire, en particulier les plus importantes, telles la Revue brésilienne d'histoire de l'ANPUH et la Revue d'histoire du département d'histoire de l'université de São Paulo, se sont montrées réceptives aux initiatives des médiévistes. Cependant, le volume des propositions reste modique, comparé à l'intérêt accru des étudiants.

Malheureusement, les traductions d'œuvres produites dans des pays connus pour leur tradition en matière d'études médiévales n'ont pas comblé cette lacune, ce qui rend particulièrement difficile le travail à déployer pour être diplômé. Les travaux de traduction sont généralement détachés des impératifs académiques et peu intéressés à former un nouveau public. Pour ces raisons, ils ont l'habitude de se concentrer sur des œuvres anciennes qui se sont déjà bien vendues hors du Brésil ainsi que sur des textes de vulgarisation, au détriment d'œuvres ayant déjà produit un impact sur la scène historiographique internationale. 
gouvernementale pour l'appui et l'évaluation des institutions n'a pas porté sur l'un des problèmes fondamentaux qui affecte la recherche: le déphasage de qualité entre l'enseignement public supérieur, les enseignements Fundamental «fondamental » : de la pré-école à la neuvième année d'études -et Médio - «moyen » : de la dixième à la quatorzième année d'études ${ }^{6}$. Ainsi, les élèves n'ont pas accès à l'ensemble des outils essentiels, indispensables dans l'enseignement supérieur, tels la maîtrise d'un cadre factuel et chronologique fondamental et une certaine expérience de la documentation et de la dimension critique de l'histoire. Tout au long de son enseignement de base, l'élève entre en contact avec l'histoire médiévale dans le cadre des études d'histoire générale - de la préhistoire au XVI ${ }^{\mathrm{e}}$ siècle -, qui, en moyenne, occupent deux heures d'enseignement par semaine, tout au long de l'année. Quant au contenu de l'histoire médiévale à proprement parler, il lui revient, en fait, entre deux et quatre heures de cours durant toute la formation scolaire pré-universitaire.

Un autre problème important, en partie perpétué par le cadre général rappelé cidessus, tient à ce que l'espace restreint réservé à l'histoire médiévale dans le cursus scolaire n'incite pas les auteurs d'ouvrages à accorder une plus grande attention aux chapitres correspondants de la période qui, outre le fait qu'ils ne sont plus d'actualité, sont remplis d'erreurs et de malentendus méthodologiques.

Cette littérature encourage normalement l'identification entre "Moyen Âge » et « féodalisme », entre seigneurie et fief, et parfois même, entre servage et vassalité, ceci sans parler de la classique opposition binaire « seigneurs » et "paysans ", si peu utile à l'éveil de la conscience sociologique des élèves. Enfin, ces livres choisissent en général de diviser le Moyen Âge en chapitres qui privilégient le Moyen Âge central et le bas Moyen Âge, souvent divisés en thèmes comme l'«Église ", le "féodalisme », la «renaissance urbaine». Ainsi, une curieuse scission s'installe dans l'esprit de nos jeunes étudiants en histoire: d'un côté, confiants dans l'autorité scolaire, ils s'imprègnent avec certitude de l'idée d'un «âge des ténèbres » et, de l'autre, de cette fantaisie médiatique autour d'un "âge des sorcières, des fées et des dragons ». Dans l'enseignement supérieur, le cours de première année sur le Moyen Âge est ainsi devenu un lieu de résolution de conflits importants - pour imposer une perspective historique scientifique, une critique des visions communes du Moyen Âge et la formation d'un répertoire analytique et conceptuel pour connaitre la période -, ce qui occupe une bonne partie du temps de cours disponible en salle de classe.

Dans l'enseignement supérieur, la structure du cursus scolaire en vigueur implique également d'importants effets sur l'étude du Moyen Âge. La majorité des universités publiques compte à peine un enseignant spécialisé responsable de l'enseignement du premier ${ }^{7}$, deuxième et troisième cycles universitaires, ainsi que de la recherche en histoire médiévale ${ }^{8}$. Seules font exception l'université de São Paulo (USP), dont le département d'histoire compte cinq spécialistes de l'histoire médiévale, l'université fédérale fluminense (UFF), qui en compte trois, et l'université fédérale de Rio de Janeiro (UFRJ), qui en comprend trois également. Dans les autres universités, le cours de formation à l'histoire médiévale dure seulement un semestre. En troisième cycle, la situation n'est pas moins difficile. Il est rare, en quelque partie du pays que ce soit, qu'un étudiant qui mène une recherche, puisse accumuler des crédits dans plus d'une des disciplines du domaine de l'histoire médiévale. Un des plus graves problèmes, qui résulte de ce contexte, tient au fait que les étudiants ont très peu de possibilités d'accéder à un corpus documentaire varié et adapté à leur formation. Il est clair que de

Bulletin du centre d'études médiévales d'Auxerre | BUCEMA, 12 | 2008 
telles difficultés ne trouveront pas de solutions gouvernementales. Nous pensons qu'elles ne peuvent être résolues que par le renforcement des groupes d'études et de recherche universitaires, de leurs demandes en matériel bibliographique aux institutions de soutien et, enfin, le développement des échanges inter-institutionnels de professionnels dotés d'une formation solide.

Dans ce contexte, les multiplicateurs de formation sont essentiels, tels ces nouveaux docteurs, qui ont accompli leurs études à l'étranger et qui ont acquis une formation que nos universités ne peuvent pas fournir et grâce à laquelle ils ont pu intégrer dans leur bagage scientifique la connaissance approfondie des archives et la maitrise des ressources techniques, telles l'étude adéquate du latin médiéval, de la paléographie, de la diplomatique et de la codicologie. Il y a encore quelques années, ces chercheurs rentraient au pays et restaient en attente de l'ouverture de concours d'accès aux universités publiques ou se résignaient à donner des cours sans rapport avec leurs compétences dans des universités privées voire même dans l'enseignement secondaire.

Le développement de la politique contractuelle, intense au cours de ces sept dernières années, touche aujourd'hui à sa fin. Les professeurs d'histoire médiévale aujourd'hui affectés aux universités publiques, âgés entre 30 et 40 ans pour la majorité d'entre eux, resteront encore 30 à 40 années de plus à leurs postes. Deux problèmes découlent de cette situation : le manque d'opportunités pour les nouvelles générations de docteurs et le "vieillissement " à long terme de ce champ d'études. Un des palliatifs à ces problèmes réside dans la possibilité, ouverte il y a quelques années, de bénéficier de bourses de post-doctorat, sur ressources provenant du gouvernement fédéral et des états fédérés. Ces bourses, attribuées à des docteurs nouvellement diplômés, leur permettent de poursuivre des activités de recherche et d'enseignement auprès des universités publiques. Ces programmes, en expansion, permettent l'absorption pour un temps déterminé de jeunes professionnels, qui contribuent non seulement à diversifier l'enseignement et la recherche, mais également à dynamiser les groupes de recherche.

Ce cadre ouvre des perspectives en vue d'étendre le champ des recherches pour ce qui concerne les thématiques, les documents et les périodes d'études. Le manque de fonds documentaires imprimés, l'éloignement des archives, les difficultés pour étudier les langues anciennes et l'insuffisance d'un nombre significatif de spécialistes orientent les recherches dans trois directions, à savoir : l'étude des $\mathrm{XIV}^{\mathrm{e}}$ et $\mathrm{XV}^{\mathrm{e}}$ siècles, l'étude du Moyen Âge portugais et le travail sur les documents littéraires.

27 Cependant, les caractéristiques de l'expérience brésilienne de recherche en histoire médiévale ne découlent pas seulement de nos carences et de nos spécificités au regard des régions européennes habituellement étudiées par les travaux médiévaux et où une bonne partie de la documentation a pu être conservée, mais également de facteurs endogènes que nous allons aborder à présent. Avant toute chose, il convient de prendre en compte le contexte dans lequel cette expérience a vu le jour ainsi que le projet fondateur des premières universités brésiliennes et, en particulier, la première d'entre elles, à savoir l'université de Sao Paulo (USP), qui, jusqu'à la fin des années 1990, était la seule à entreprendre des études doctorales et des recherches en histoire médiévale.

L'USP fut l'un des produits de la transformation de l'élite pauliste au début du $\mathrm{XX}^{\mathrm{e}}$ siècle, générée par la richesse découlant du café, puis de l'industrialisation. Celle-ci eut des répercussions importantes dans le champ culturel, illustrées notamment par la Semaine d'art moderne en 1922. Cette université fit partie du projet culturel orienté vers la production et le maintien de l'hégémonie politique de l'État de São Paulo dans le 
scénario national. Constituée à partir du noyau préexistant des facultés de droit, de médecine et de l'école polytechnique, l'université s'est cependant structurée autour de la Faculté de philosophie, des sciences et des lettres, en privilégiant les domaines fondamentaux du savoir - la philosophie, l'histoire, la physique, les mathématiques et les lettres.

Jusqu'alors, la recherche en histoire était concentrée à l'IHGB (Institut historique et géographique du Brésil), fondé en 1838, et voué au développement de ce qu'on pourrait qualifier d'histoire nationale. L'université de São Paulo, créée en 1934, choisit de suivre d'autres chemins, éloignés tant du nationalisme, cher à la dictature de Vargas, que du régionalisme, préférant adhérer à l'idéal de civilisation européen. Des professeurs étrangers furent invités pour aider aux premiers pas de l'implantation du projet universitaire. C'est ainsi que furent accueillies des «missions » de chercheurs italiens, allemands et français.

Ces missions bénéficièrent de la jeunesse de certains chercheurs alors à l'apogée de leur créativité. Pour ce qui est des Français, sont passés par l'USP : Fernand Braudel, Claude Lévi-Strauss, Roger Bastide, Émile Coornaert, Émile Léonard, Jean Gagé et Pierre Monbeig. Jean Gagé, spécialiste de l'histoire de Rome, y dirigea les premières recherches sur l'histoire médiévale de notre pays. En 1942, Eurípides Simões de Paula, assistant de la chaire d'« Histoire des civilisations » obtint, sous la direction de Gagé, le premier doctorat d'histoire présenté au Brésil, ayant pour thème Le commerce varègue dans la principauté de Kiev au XII siècle - "O comercio varegue e o Grão-Principado de Kiev ». En 1945, Pedro Moacyr Campos, futur professeur d'histoire médiévale de l'université, défendit sa thèse sur : Alguns aspectos da antiga Germânia através dos autores clássicos - Certains aspects de la Germanie antique, à travers les auteurs classiques.

Plusieurs tendances concernant ces études, et qui resteront d'actualité durant de nombreuses décennies, se firent alors jour: l'absence de constitution d'un corps de directeurs d'études spécialisés et l'affectation d'enseignants dont le champ de spécialisation se situait ailleurs, comme ce fut le cas de Pedro Moacyr Campos, spécialiste en lettres germaniques. Axé sur la conception " civilisationnelle », Eurípides Simões de Paula a dirigé des thèses portant sur des thèmes de l'histoire antique l'économie, la question agraire et le judaïsme -, de l'histoire médiévale - sur les relations entre Byzance et la papauté, la législation économique et sociale à l'époque de la peste noire, les relations entre juifs et Russes, la culture judaïque, la formation de l'Angleterre, l'histoire ibérique -, mais aussi sur la théologie, le bouddhisme, les théories scientifiques au XVI ${ }^{e}$ siècle, la langue et la littérature en Russie, en Arménie, en Chine et dans le monde musulman ${ }^{9}$.

Ce cadre général met en évidence la cohérence de conception du cours sur le concept de «civilisation" - plus tard l'étude du Moyen Âge apparaîtra dans le cadre des disciplines dispensées en cours d'histoire sous le nom de "Civilisation antique et médiévale » -, l'immense liberté des directeurs d'études, dont la production pouvait correspondre aux intérêts particuliers de leur érudition et de leur savoir, et l'indépendance des chaires d'enseignement, dont l'engagement n'était pas associé au développement d'un champ précis de savoir. Ces premières générations d'enseignants n'étaient donc pas engagées dans un projet de mise en valeur et de structuration des secteurs de la connaissance historique, ce qui ne surviendra qu'à partir des années 1980. Les relations personnelles déterminaient le plus souvent les lignes de recherche, voire même l'accès au matériel d'études, étant donné que la mise à jour des 
bibliothèques universitaires n'était pas, jusqu'il y a peu, une priorité pour de nombreux enseignants. De sorte que les élèves dépendaient beaucoup des bibliothèques particulières de leurs directeurs d'études. Ainsi, le doctorat d'Euripides Simões de Paula ne présageait-il en rien l'avenir de son domaine d'études, mais restait lié au projet commun de réflexion sur le fait civilisationnel.

En dépit du pouvoir isolé des professeurs titulaires de chaires, la multiplicité de leur centre d'intérêts a fonctionné comme un facteur de dispersion bénéfique au secteur. Ainsi, le paradigme du " génie fondateur ", de l'arbre mythique dont proviennent tous les fruits, qui est valable pour l'histoire des études médiévales de certains pays, ne l'est pas au Brésil. Ce champ d'études s'est formé en carence de fondateurs de hiérarchies claires, propres à se reproduire après eux. Par ailleurs, l'Université s'est édifiée à contresens du centralisme fédéral des années 1930 et par la suite s'est montrée défiante, dans son domaine d'activité, envers certaines formes d'organisation du pouvoir. Le système de partenariat avec des pays étrangers lui a permis de préserver son goût de l'équité. Ainsi, l'idée de "civilisation", la critique du centralisme et le manque de spécialisation stricte et de mise en valeur des aires du savoir ont empêché l'apparition et l'imposition d'une personnalité capable d'établir un projet historiographique pour les études médiévales qui aurait pu devenir à long terme hégémonique, préservant ainsi le pluralisme.

L'histoire du Moyen Âge, qui commence à s'esquisser au Brésil, n'est pas une histoire auto-référente. Ça ne l'est pas dans la mesure où elle ne dépend pas des destinées strictes de l'historiographie ibérique, encore qu'elle s'en tienne en priorité aux documents ibériques. Mais elle le fait toujours à partir des fondements théoricométhodologiques qui échappent à la péninsule. Ces études, à leur tour, en dépit de leur intérêt significatif pour l'historiographie française, ne sont pas de nos jours limitées à cette influence. Au contraire, notre attachement au dialogue inter-historiographique, notre intérêt pour les divers courants historiographiques et la critique de leurs limites nationales apparaissent chaque fois de façon plus évidente.

Le retard pris par le projet d'implantation des universités au Brésil en comparaison avec les pays d'Amérique de langue espagnole a permis que celles-ci apparaissent en plein milieu de la vague moderniste ${ }^{10}$, garantissant du même coup à ces institutions une plus grande liberté au regard de l'idéologie nationaliste et de l'influence cléricale. Deux éléments positifs en découlent. En premier lieu, à la différence de l'historiographie européenne proprement dite, «notre » Moyen Âge fait abstraction du problème national. En second lieu, ce Moyen Âge se distancie clairement de l'idéal de construction européenne qui oriente aujourd'hui une grande partie des études européennes.

Je ne pense pas que nous puissions déjà proposer des résultats consistants dans le sens d'une contribution historiographique découlant de ces spécificités, mais il est certain que, à condition que se poursuivent les projets d'amélioration de nos conditions de travail, cela se produira. Une conscience accrue des besoins institutionnels et des responsabilités individuelles pour le développement de notre domaine d'activité va dans cette direction. 


\section{BIBLIOGRAPHIE}

J. JOBSON DE ANDRADE ARRUDA, « Linhagens historiográficas contemporâneas : por uma nova síntese histórica ", in V. LEITE FRÓES (coord.), Viagens, viajantes, almocreves, bandeirantes, tropeiros e navegantes, III colóquio luso-brasileiro, Niterói/Rio de Janeiro/Foz do Iguaçu, FAPERJ, 1996, t. 2, p. 11-23.

C. Astarita, «La historia social y el medievalismo argentino », Bulletin du Centre d'études médiévales d'Auxerre, 7 (2003), p. 113-124.

M. CANDIDO DA SILVA, « Les études en histoire médiévale au Brésil : bilan et perspectives », publication électronique : « http://ciham.ish-lyon.cnrs.fr/Brazil.html ».

M. H. R. CAPelato, R. Glezer et V. L. A. Ferlini, « Escola Uspiana de História », Estudos Avançados, 22 (1994), p. 349-358.

M. H. R. CAPELATo (coord.), Produção histórica no Brasil (1985-1994), São Paulo, CNPq/História USP/ ANPUH, 1995, 3 vol.

M. I. CARzolio, «L'histoire du Moyen Âge en Argentine : Claudio Sánchez Albornoz et ses disciples », Bulletin du Centre d'études médiévales d'Auxerre, 7 (2003), p. 99-112.

CNPq, Avaliações e perspectivas, 1977 et 1982.

F. J. CALAZANS FALCON, « Historiografia brasileira : alguns problemas e perspectivas », in V. LeITE FRÓES (coord.), Viagens..., op. cit., p. 231-245.

C. Fico et R. Polito, A história no Brasil (1980-1989), Ouro Preto, UFOP, 1992.

J. R. A. LAPA, A história em questão (Historiografia brasileira contemporânea), Petrópolis, Vozes, 1976.

J. R. A. LAPA, História e historiografia do Brasil pós 64, Rio de Janeiro, Paz e Terra, 1985.

F. IGLÉSIAS, Historiadores do Brasil, Rio de Janeiro/Belo Horizonte, Nova Fronteira/UFMG, 2000.

J. R. MACEDo (éd.), Os estudos medievais no Brasil. Catálogo de Dissertações e Teses : Filosofia, História e Letras (1990-2002), Porto Alegre, UFRGS, 2003.

J. R. MACEDO, « Os estudos medievais no Brasil : tentativa de síntese », publication électronique : «http://www.storia.unifi.it/_RM/rivista/dwnl/Rivair\%20Macedo.pdf ».

P. H. MARTINEZ, « Fernand Braudel e a primeira geração de historiadores universitários da USP (1935-1956) », Revista de História, 146 (2002), p. 11-27.

MEC-SESU, Diagnóstico e avaliação dos cursos de História no Brasil.

S. Mотоунам, Fapesp : Uma História de Política Científica e Tecnológica e Para uma História da Fapesp. Marcos Documentais, São Paulo, Fapesp, 1999.

M. J. motta Bastos et H. Franco JR, « L'histoire du Moyen Âge au Brésil », Bulletin du Centre d'études médiévales d'Auxerre, 7 (2003), p. 125-131.

P. PETITJEAN et M. PATY, « Sur l'influence scientifique française au Brésil aux XIX et $\mathrm{XX}^{\mathrm{e}}$ siècles », Cahiers des Amériques Latines, 4 (1985).

USP, « Dissertações e teses defendidas na FFLCH/USP - 1939-1977 », USP - Bol. Esp., 1, 1977.

\section{Sites internet :}


http://www.capes.gov.br/sobre/estatisticas/

http://www.capes.gov.br/sobre/historia.html

http://www.comciencia.br/resenhas/fapesp.htm

http://www.fapesp.br/materia.php?data[id_materia] $=485$

http://www.iesalc.unesco.org.ve/programas/postgrados/Informe\%20Postgrados\%20-

\%20Brasil.pdf

http://memoria.cnpq.br/sobrecnpq/historia.htm

\section{NOTES}

*. Recherche réalisée avec l'appui de la Fundação de Amparo à Pesquisa do Estado de São Paulo (FAPESP, Fondation de soutien à la recherche de l'État de São Paulo).

2. NT : en portugais, "mestrado", grade universitaire brésilien correspondant à une scolarité « Bac +6 » en France, c'est-à-dire, pouvant correspondre au niveau « master » actuel, terme que nous utiliserons dorénavant pour désigner le « mestrado ».

3. Le dernier « Fap-livres » réalisé en 2005 a acquis 7232 volumes d'histoire, de philosophie et de littérature médiévale, concernant notamment des études et des documents pour les instituts de sciences humaines de l'Université de São Paulo (USP) et de l'université d'État de Campinas (UNICAMP), dont les départements d'histoire médiévale développent des activités associées, par l'intermédiaire du Laboratório de Estudos Medievais (Laboratoire d'études médiévales, LEME/USPUNICAMP). Le coût total des acquisitions concernant les études médiévales des deux universités s'est élevé, en cette occasion, à près de 480000 euros.

4. J. R. MACEDO, «Os estudos medievais no Brasil : tentativa de síntese » [Les études médiévales au Brésil : essai de synthèse], publication électronique : «http://www.storia.unifi.it/_RM/rivista/ dwnl/Rivair\%20Macedo.pdf ».

5. « Dualité de juridiction et subjectivité politique dans l'Occident médiéval. »

6. L'enseignement «fondamental» au Brésil comprend dix années d'études et pourrait correspondre en France à la période allant du cours préparatoire au collège, en passant par le primaire. L'enseignement "moyen» comprend quatre années d'études et correspondrait en France aux années de lycée.

7. De nombreuses universités publiques ne comptent encore que sur des professeurs d'histoire médiévale non spécialisés, c'est-à-dire, des docteurs qui produisent et orientent des recherches dans un autre domaine de connaissance.

8. De manière générale, dans les universités privées, la discipline n'est pas enseignée par un docteur en histoire médiévale.

9. M. CANDIDO DA SILVA, "Les études en histoire médiévale au Brésil : bilan et perspectives", publication électronique : «http://ciham.ish-lyon.cnrs.fr/Brazil.html».

10. Tandis que l'université s'édifie au Brésil en 1934, dans l'Amérique de langue espagnole elle a accompagné le processus de conquête européenne: université de Saint-Domingue, en 1538; université San Marcos (Pérou), en 1551 ; et université du Mexique, en 1553. Le même phénomène s'est produit aux États-Unis, mais selon un processus politique inverse à l'Amérique espagnole. C'est à cause du désintérêt de la couronne anglaise pour ses colonies d'Amérique que les colons décident de fonder en 1636 l'université de Harvard. 


\section{AUTEUR}

NÉRI DE BARROS ALMEIDA

Université d'État de Campinas (UNICAMP, Brésil) 